\title{
THE ROLE OF DUPLICATIONS IN EVOLUTION
}

\author{
O. MAYO \\ Department of Human Genetics, University of Edinburgh
}

Received 30.xii.69

\section{INTRODUGTION}

Duplication of genetic material has long been regarded as a fruitful source of raw material for evolution (Haldane, 1932; Bridges, 1935). Stephens (1951), in reviewing its possible significance, felt that, to demonstrate its importance, the existence of loci of duplicate origin performing different functions must be proved. This, he suggested, required that they meet three criteria: there must be at least two distinct loci involved; their origin from one other locus or region of the genetic material must be established; and they must be shown to have different functions. These requirements are particularly stringent (for example, the second cannot be proved $a$ posteriori, but can only be treated probabilistically) and it is perhaps not surprising that Stephens felt that there was no observed case satisfying all three.

However, recently it has been shown, on the one hand, that there are long repeated sequences in the DNA of higher organisms, considered by some to be attributable to common origins, i.e. duplication, and on the other hand that some proteins are made up of very similar subunits produced by different genes (Ingram, 1961; Smithies, 1962). Furthermore, Kimura $(1968 a, b)$ has made calculations which suggest that there may be very high rates of neutral mutation of the base-pair replacement type and hence of basepair substitution, so that duplication may be more important than previously thought in making substantial changes unlikely to be neutral. It may be that Kimura's arguments overstate these mutation rates, but even so, it is clear that one can now consider whether there are duplications fulfilling Stephens's requirements for a demonstration of their evolutionary significance.

But more important, perhaps, are the consequences of the model of genic regulation in higher organisms advanced by Britten and Davidson (1969). They suggest that " batteries" of "producer" (structural) genes are regulated by "activator" RNA synthesised on "integrator" genes, whose effect is " to induce transcription of many producer genes in response to a single molecular event". Since the evolutionary consequences of this model are evidently different from those of the model of evolution by repeated duplication proposed by Nei (1969), and this in turn differs from that of Watts and Watts (1968), there is perhaps merit in a further reexamination of the role of duplication in evolution.

\section{The aGtion of SELEGTION ON DUPLIGATIONS}

Gene duplication can occur in at least three ways: by unequal crossingover, by regional redundant duplication and by polyploidy (Ohno, Muramoto, Klein and Atkin, 1969). Here, we are in general concerned with the first two kinds. It is difficult to postulate simple, convincing cytological 
models for the occurrence of a primary duplication (Watts and Watts, 1968), particularly with the restriction that the duplication should be interstitial (see e.g. Whitehouse, 1965). However, this partly supposes that the meiotic and mitotic mechanisms have always been as they are now, whereas some duplications may be very old (Ohno et al., 1969) and the cytological mechanisms must have also evolved.

Watts and Watts (1968) have emphasised that structural homology, possessed at most sites by allelomorphs, is not necessarily the same as locational homology, required for proper pairing. Thus, they ask of a region of genetic material, "what percentage of the base pairs can be different without lowering the degree of homology so far as to interfere with the capacity for crossing-over?" They point out that the $\alpha$ and $\beta$ haemoglobin chains have 146 amino acid residues and differ in a minimum of 2.55 per cent. of base pairs, ignoring redundancy, and there are thus three long regions where they can be completely homologous, and this is where the cross-overs in known Lepore haemoglobins occur (Baglioni, 1968).

This is mentioned here, rather than in the next section, since it suggests a possible selective mechanism acting by means of mutations which may be occurring very frequently (Kimura, 1968a, $b, 1969$ ). Thus, two duplicate loci which had accumulated a number of random, nearly neutral mutations would be less likely after unequal crossing-over to make a functional product than would two nearly identical duplicate loci, like the $H b \beta$ and $H b \delta$ loci. Thus, the accumulation of nearly neutral mutations might restrict the frequency of unequal crossing-over.

However, Watts and Watts (1968) emphasise that " mechanisms which protected organisms from drift towards a randomisation of synonymous codons would have developed during evolution ", even though, as they add, a single mutation of a codon to a synonym cannot be selected at the level of its product, and many non-synonymous mutations appear to have little effect in heterozygotes; and they conclude that lessening of homology between alleles will be directly or indirectly disadvantageous. A suggestion of non-randomness comes from Kimura (1968b), who has calculated the expected proportions of various amino acids in the proteins of vertebrates, assuming among other things complete randomness of synonyms, and has found that there is not very good agreement with observed values. Of course, such calculations are as yet inaccurate, other aspects of the selection acting on chromosomal abnormalities can be quantified more precisely.

A duplication is a repeated sequence of genetic material, not necessarily found contiguous to the sequence from which it arose, nor even in the same chromosome. Watts and Watts (1968) have considered the cytological mechanisms whereby it may arise. If in the same chromosome and contiguous to its parent sequence, it may be in the same order as its parent (tandem repeat (Bridges, 1935)) or reversed. These different positions may be of immediate genetical significance but phenotypic changes associated purely with structural alteration are in general unstable (Lewis, 1950), so they can be ignored, though they may be important in Britten and Davidson's (1969) theory; this is not clear. The fact that an inversion suppresses crossing-over may, however, be important. Metz (1947) has suggested that there are three criteria for a duplication to be important in evolution: it must be small, beneficial and sufficiently stable to persist. Since duplications arise by unequal crossing-over (cf. Catcheside, 1947), approximate 
reversions can readily occur for tandem repeats, and Metz pointed out that reverse repeats were more commonly found in natural populations.

If a duplication has an advantage, this may be one of a variety of kinds: it may produce more of a given product, or products, depending on how many functional loci it contains, and this may lead to epistasis; it may allow evolution of a new gene product, since the parent gene can produce the original product; or it may produce a position effect. The first two kinds of advantage can readily lead to fixation of the material or, on occasion, to polymorphism.

In some ways, then, a duplication is very similar to an inversion. Indeed, it may well be an inversion, though this is not true for several cases of interest, as discussed below. Haldane (1957) and Kojima (Nei, Kojima and Schaffer, 1964; Kojima, 1967; Ohta and Kojima, 1968) have presented conditions for the generation and maintenance of inversion polymorphisms, which will be applicable to any region, containing at least one locus which is not undergoing much recombination.

Kojima (Nei et al., 1967) has shown, by arguments which follow closely those of Fisher (1930) for a single locus, that if such a region contained many dominant favourable alleles, it could increase very rapidly in frequency if these were initially rare. In general, such obviously favourable conditions are unlikely to be met.

For an infinite population, Ohta and Kojima (1968) have established the criterion for ultimate extinction of an inversion to be $\prod_{t=0}^{\infty} C_{t}<\infty$ when $C_{t}$ is the mean number of inversion heterozygote progeny per inversion heterozygote at generation $t$ after the occurrence of the inversion. More simply, this is essentially saying that the mean number of inversion heterozygote progeny per inversion heterozygote over all generations must be less than one, as for any Galton-Watson process (Feller, 1957, p. 276). The same condition will apply to any other chromosomal aberration. Ohta and Kojima further derive the ultimate survival probability of an inversion, which carries a unique advantage, since, from the argument above, any chromosomal segment not having such an advantage will be lost. As expected, the results are essentially comparable with those for a single locus (Fisher, 1930), e.g. if a single gene has an additive advantage $\frac{1}{2} s$ in the heterozygote, its ultimate survival probability (chance of fixation) is $s$. Ignoring complications from unequal crossing-over, these results may be applied to duplications.

Spofford (1969) has indeed elegantly presented the appropriate calculations for the process of incorporation of duplications into a population, using, as one would expect, the ordinary methods of population genetics. A number of assumptions were made in her model which may not always hold. For example, that the duplicated region is a complete cistron. As will be discussed further below, this is a reasonable assumption, but detracts from the generality of her results, though in a conservative way.

She has shown that "single locus heterosis is sufficient for the incorporation of a newly arisen duplication into a species", such heterosis assumed to arise "from the formation of heterodimers between polypeptides specified by alleles ". (See Schwartz and Laughner (1969) for a possible example of such heterosis, in the alcohol dehydrogenase isozyme polymorphism in maize.) 
With Spofford's extensive demonstration in mind, then, one can consider, in a very simple way, the number of newly arising duplications which are absorbed into a population. Spofford has accepted that an initial advantage is almost essential for the absorption of a new duplication, but the importance of the advantage depends on the frequency with which the duplications occur: this is because neutral mutants are fixed at approximately the same rate as they occur (Kimura, 1968a).

Accordingly, suppose that these are $n$ sites at which a duplication can occur, which for a duplication to be neutral or advantageous may well be of the order of the number of cistrons in the genome, since duplications starting or ending within a cistron appear likely to be deleterious (though this

TABLE 1

Chance, $\mathrm{P}$, of one or more duplications being fixed per gensration in a population of size $\mathrm{N}$, together with the mean number, $\mathrm{m}$ of duplications fixed per generation. Here, $\mathbf{n}$ represents the number of sites per genome at which duplications can occur, $\mu$ is the rate per site of occurrence of new duplications, and $\mathrm{s}$ is the selective advantage of each new duplication

\begin{tabular}{|c|c|c|c|c|c|}
\hline$\mu$ & $n$ & $\mathcal{N}$ & $s$ & $P$ & $m$ \\
\hline \multirow[t]{5}{*}{$10^{-7}$} & $5 \cdot 10^{5}$ & $10^{8}$ & 0 & 0.0241 & 0.0244 \\
\hline & & & $10^{-4}$ & 0.0291 & 0.0296 \\
\hline & & $10^{4}$ & $\begin{array}{l}0 \\
10^{-4}\end{array}$ & $\begin{array}{l}0.0241 \\
0.0946\end{array}$ & $\begin{array}{l}0.0244 \\
0.0994\end{array}$ \\
\hline & $10^{8}$ & $10^{3}$ & $\begin{array}{l}0 \\
10^{-4}\end{array}$ & $\begin{array}{l}0.0465 \\
0.0561\end{array}$ & $\begin{array}{l}0.0476 \\
0.0577\end{array}$ \\
\hline & & $10^{4}$ & $\begin{array}{l}0 \\
10-4\end{array}$ & $\begin{array}{l}0.0465 \\
0.0561\end{array}$ & $\begin{array}{l}0.0476 \\
0.0577\end{array}$ \\
\hline \multirow[t]{4}{*}{$10^{-8}$} & $5 \cdot 10^{5}$ & $10^{3}$ & $\begin{array}{l}0 \\
10^{-4}\end{array}$ & $\begin{array}{l}0.0025 \\
0.0030\end{array}$ & $\begin{array}{l}0.0025 \\
0.0030\end{array}$ \\
\hline & & $10^{4}$ & $\begin{array}{l}0 \\
10^{-4}\end{array}$ & $\begin{array}{l}0.0025 \\
0.0101\end{array}$ & $\begin{array}{l}0 \cdot 0025 \\
0 \cdot 0102\end{array}$ \\
\hline & $10^{6}$ & $10^{3}$ & $\begin{array}{l}0 \\
10^{-4}\end{array}$ & $\begin{array}{l}0 \cdot 0050 \\
0.0060\end{array}$ & $\begin{array}{l}0 \cdot 0050 \\
0 \cdot 0060\end{array}$ \\
\hline & & $10^{4}$ & $\begin{array}{l}0 \\
10-4\end{array}$ & $\begin{array}{l}0.0050 \\
0.0201\end{array}$ & $\begin{array}{l}0.0050 \\
0.0203\end{array}$ \\
\hline
\end{tabular}

need not be the case; see section 3 ). The proportion of zygotes carrying a new duplication each generation will be $p_{1}=1-(1-\mu)^{2 n}$, where $\mu$ is the rate, per possible site, of occurrence of new duplication. Now let the chance of a duplication being fixed be $p_{2}$. For a neutral mutant gene Fisher (1930) has shown $p_{2}=\frac{1}{2 \mathcal{N}}$ in a population of size $\mathcal{N}$, and if the mutant has selective advantage $s, p_{2}=\frac{1-e^{-28}}{1-e^{-4 N_{8}}}$ (it is assumed that new duplications act like mutant genes). For any individual, the chance that it has a duplication which is fixed is $p_{1} p_{2}$. The chance of at least one duplication being fixed per generation is thus $P=1-P_{o}, P_{o}=\left(1-p_{1} p_{2}\right)^{N}$. From a Poisson distribution with $P_{o}=e^{-m}$, the mean number, $m$ of new duplications fixed per generation can be calculated. Some results are shown in table 1.

This argument clearly neglects many important factors, but it does indicate that, even assuming duplications to be very rare events, their rates of incorporation are not negligible. 
Once a duplication is established, further change depends on its nature. If it is translocated or inverted, reversion or other change by unequal crossing-over is unlikely, but mutation, followed by natural selection and drift, will allow separate evolution of the two units. If it is a tandem repeat, however, reversion and multiplication can occur by unequal but homologous crossing-over (Sturtevant, 1925; Metz, 1957; Smithies, 1964). Sturtevant (1925) observed, at the Bar locus, 8 out of 20,439 changes from $B$ to " double Bar". This duplication is quite a substantial one (Bridges, 1935), and it seems reasonable that the chance of unequal crossing-over rises with the length of the duplication. Accordingly, one can say that a very high frequency of unequal crossing-over is unlikely to be more than a few per cent.

Ignoring mutation, one can derive approximate frequencies for reversion as follows. Suppose $A$ to be the original " allele " and $A^{\prime}$ the duplication, with a rate of $r$ of unequal crossing over in $A^{\prime} A^{\prime}$ to give $A$ and $A^{\prime \prime}$. Then assuming that $A^{\prime \prime}$ is not advantageous compared with $A$, and with these fitnesses:

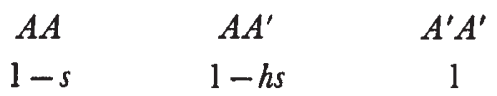

the frequency of $A$ will be approximately $\frac{r}{2 h s}$. If $A$ is recessive to $A^{\prime}$, i.e. with fitnesses:

$\begin{array}{ccc}A A & A A^{\prime} & A^{\prime} A^{\prime} \\ 1-s & 1 & 1\end{array}$

then its frequency will be approximately $\frac{r}{2 s}$.

These results, of course, apply only in an infinite population, and in the case where triplications, etc., which are produced whenever revertants occur, are not lethal (a possible example is pig plasma aryl esterase (Lush, 1966): see next section) the situation will be more complex. In a population of size 500, with the techniques of genetical simulation of Mayo (1967), the results in table 2 were obtained.

As indicated in table 2, models were used both with triplications, etc., lethal and without, in the latter case having fitnesses symmetrical about the peak genotype, $2 / 2$, where 1 was one dose, 2 two and so on.

Clearly, as one expects, the frequencies of 1,3 , etc., are low unless the selection against them is very weak or the frequency of homologous, unequal crossing-over is very high.

Haldane (1957) showed that, for stability of a chromosome polymorphism, heterozygotes at any locus concerned in the genetical polymorphism should have greater effect when other such loci are heterozygous than when they are homozygous. However, for an inversion containing a sex-determining mechanism, only one advantageous gene is needed, which, Haldane felt, accounted "for the fact that structural polymorphism is much more commoner in sex-determining chromosome than in autosomes". However, some of this polymorphism may be associated with peculiarities in the evolution of the sex chromosomes, which may have evolved heterogamy as a mechanism for sex determination, and with the fact that complete synapsis 
is not required for the sex chromosomes in the heterogametic sex. Thus, one cannot make the plausible prediction that duplicate loci should be more common on the sex chromosome. (Furthermore, if one excludes sexdetermining heterogamy, there is not much evidence of greater structural polymorphism in the sex chromosomes.)

TABLE 2

Frequency of a disadvantageous gene produced by unequal homologous crossing-over from an advantageous duplication. The initial frequency of the gene was $0.01 ; \mathrm{q}$ is the mean frequency for 50 successive generations, $\mathrm{r}$ is the frequency of crossing-over, and $\mathrm{s}$ is the intensity of selection

$\begin{array}{cccl}r & & & \begin{array}{c}\text { Sampling } \\ \text { variance } \\ \text { of } q\end{array} \\ 0.01 & s & \bar{q} & 0.00120 \\ & 0.05^{*} & 0.11 & 0.000624 \\ & 0.05 \dagger & 0.080 & 0.000193 \\ & 0.10^{*} & 0.051 & 0.000421 \\ 0.001 & 0.10 \dagger & 0.099 & \\ & 0.05^{*} & 0.019 & 0.000102 \\ & 0.05 \dagger & 0.021 & 0.0000454 \\ & 0.10^{*} & 0.0060 & 0.00000981 \\ & 0.10 \dagger & 0.0067 & 0.0000179\end{array}$

* Selection increased additively by $\frac{1}{2} s$ as genotypes diverge from $2 / 2$.

$\dagger$ Selection increased additively by $\frac{1}{2} s$ as genotypes diverge from $2 / 2$ for losses of genetic material $(1 / 1,1 / 2$, etc.); triplications, etc., lethal.

In developing their model of regulation, Britten and Davidson (1969) emphasised very strongly that " the likelihood of utilisation of new DNA for regulation is . . . far greater than the likelihood of invention of a new and useful amino acid sequence (following a saltatory mitation)", essentially since an integrated system would require too many coincident events. But in the case of the duplication of an existing structural gene, this means that the new gene will not be functional, particularly in view of the further requirement of Britten and Davidson that there be a receptor gene, which will activate the producer, contiguous with the producer. (This is not so for the case of duplication of a complete chromosome or genome.) Thus, if Britten's model is correct, the views elaborated above about selection of duplications may require extensive modification.

\section{The Results of Duplication}

It may well be rare for an enzyme to be a monomer (Zimmerman and Gundelach, 1969) and several have been found to be made up of multiples of two polypeptide chains (Lush, 1966). The finding that these chains can be very similar, but are evidently produced by different gene loci, has led to the suggestion that these particular loci have arisen by duplication (Ingram, 1961; Smithies, 1962; Lush, 1966).

Lush (1966) refers to about ten such cases among eighty or more polymorphisms discussed in vertebrates other than man, including that of the enzyme plasma aryl esterase in the pig, where the alleles appear to be zero, one, two, three or four doses of a basic gene. However, the two proteins most closely examined are haptoglobin (Smithies, 1962, 1964) and haemoglobin (Ingram, 1961; Nance, 1963). 
Haptoglobin is a plasma protein with the ability to bind haemoglobin, so it is particularly important, for example, in haemolytic disease (e.g. Fraser, Steinberg, Defaranas, Mayo, Stamatoyannopoulos and Motulsky, 1969). There are three common alleles at the locus determining its $\alpha$-chain, $H p^{1 F}, H p^{1 S}$ and $H p^{2}$, and Smithies $(1962,1964)$ suggested that alpha polypeptide $h p 2$ (determined by $H p^{2}$ ) was produced by a partial gene duplication, arising from a unique unequal cross-over in an $H p^{1 F} / H p^{1 S}$ heterozygote, since the sequence of amino acids in the $h p l \alpha$ polypeptide occurred essentially twice in the $h p 2 \alpha$ polypeptide which was almost twice as long as the other. Additionally, Haptoglobin Johnson, produced by $H p^{2 J}$, appeared to be a triplication, and prediction of the properties of products of unequal crossing-over appeared to be confirmed (Smithies, 1964).

Smithies (1964) pointed out that one of the reactions occurring in the degradation of haemoglobin to biliverdin had been shown to occur more rapidly when the haemoglobin was bound to haptoglobin from $H p^{2} / H p^{2}$ than from the other genotypes (Nakajima, Takemura, Nakajima and Yamaoka, 1963), which might provide a basis for selection for the allele $H p^{2}$. He then suggested, following Nance (1963), that this would never lead to fixation of $H p^{2}$, because $H p^{1}$ would continually be found by unequal but homologous crossing-over. However, $H p^{1}$ has frequencies as high as $0 \cdot 7$ in regions of South America where haemolytic disease is not very frequent (Neel, Salzano, Junqueira, Keiter and Maybury-Lewis, 1964), even though it is regarded as only being at an advantage, from its greater haemoglobinbinding ability, when such disease is frequent (Baxi and Camoens, 1969). Workman (1968) has presented evidence that there is selection against $H p^{1}$, particularly $\mathrm{H} p^{1 F}$, in Negroes in the U.S.A.; the frequency of this allele was high originally, it has been proposed, since it improved survival chances in haemolytic disease arising from the polymorphisms conferring protection against malaria (Allison, 1964).

Even considering $H p^{2}$ frequencies as high as 0.6-0.8 in other populations (Workman, 1968; Baxi and Camoens, 1969) it is clear that there is no equilibrium between selection for $H p^{2}$ and recombination yielding $H p^{1}$, since, from the approximate relations $q=\frac{r}{2 h s}$ or $q=\frac{r}{2 s}$, widely varying $r$ and $s$ values would be needed in different populations, and in general $r$ would probably not be high enough to cope with anything but very weak selection. A combination of various selective forces, observed or possibly not yet detected, seems a more likely explanation of the polymorphism.

Smithies $(1962,1969)$ has emphasised the way that partial gene duplication can lead to production of a continuous, uninterrupted polypeptide covering the beginning of the duplication, so that it is distinct from the original polypeptide immediately, enabling the bypass of the possibly very slow process of random mutation followed by selection of mutant types. However, in the case of haemoglobin, Ingram (1961) has emphasised that at least two complete duplications, and possibly a third, have occurred, to produce the $\alpha, \beta, \gamma$ and $\delta$ chains, since these are all about the same length (140 amino acid residues) and differ, for example in man, from one another in about 10 to 50 . Postulates required to explain the present nature of haemoglobin are as follows: as already mentioned, there have been several duplications, followed or accompanied by translocation (so that the $\beta$ and $\delta$ loci are linked, but the others are independent); when the chains form a 
tetramer $\alpha_{2} \beta_{2}$ or $\alpha_{2} \gamma_{2}, \alpha$ cannot undergo so much evolutionary change (thalassaemia and sickle cell anaemia are $\beta$-chain abnormalities); and the time of change has been from $\alpha$ to $\gamma$ to $\beta$ to $\delta$. The second postulate perhaps anticipates Kimura's (1969) recent findings of different constant rates of change in the $\alpha$ and $\beta$ chains throughout mammalian evolution. In general, results obtained support the hypothesis (Zuckerkandel and Pauling, 1965).

Nance (1963), in considering the evolution of the haemoglobins, pointed to the possibility that, by example chance loss of an inversion, a duplication could become unstable, if it was still a tandem repeat, so that, perhaps, the " high frequency of thalassemia in certain populations may not invariably result from peculiarities in the environment". However, since thalassaemia is now known to be caused by complete or partial inactivation of the synthesis of the $\alpha$ - or $\beta$-chain, rather than by a incorrectly coded chain (Weatherall, 1969), this suggestion is clearly incorrect. Furthermore, he suggested that, for the sick cell trait, $H b \beta A \beta S$ " alleles" could have arisen on a single chromosome by unequal crossing-over, giving the protection against malaria enjoyed by the $H b \beta A / H b \beta S$ heterozygote without the possibility of forming lethal $H b \beta S / H b \beta S$ zygotes. Thus if the frequency of the sickle cell trait (heterozygote) is $x$ at birth, the frequency of sickle cell anaemia at birth is much less than $\frac{(1-\sqrt{1-2 x})^{2}}{4}$. This, he said, was the case, but Allison (1964), in disagreeing, pointed to later work showing agreement with expectation and also to the absence of $H b-A$ from many cases of $H b-A$ from many cases of $H b \beta S / H b \beta^{C}$. However, there does seem to be agreement that some unequal crossing over does occur.

\section{Discussion}

Nance (1963) has extended the potentiality of the breakdown of duplication to provide " an attractive supplement to selection genetic drift and inbreeding as possible explanations for the extraordinary frequency of the genes for kuru among the Fore ..., for amyotrophic lateral sclerosis among the Chamorros ...., for albinism among the Cuna Indians, and perhaps even for the prevalence of diabetes and cystic fibrosis among Caucasians". This is certainly an extreme use of the concept of duplication (for example, kuru is no longer thought to be genetically determined (e.g. Rogers, Barsnight, Gibbs and Gajdusek, 1967)), but it is clear that the role of duplication in evolution is not simply to provide raw material for evolution. Considering evolution as the accumulation of information (Kimura, 1961), duplication offers a number of ways of increasing the rate of the process. It can lead immediately to new gene products (Smithies, 1964); it can allow changes at one locus while a still advantageous point is made at the other (Ingram, 1961); it can increase the rate of production of particular products and their polymers (Hopkinson, Spencer and Harris, 1964; Smithies, 1964); and it can probably alter dominance relationships without the intervention of selection (Zimmerman and Gundelach, 1968).

This assumes that duplications have occurred, since the chance of two very similar polypeptides arising independently is particularly low (Smithies, 1964), though such convergence could occur, and duplication provides the best explanation of such observations. Thus it may be said that, in so far as 
they can be met a posteriori, Stephens's (1951) criteria for the demonstration of the occurrence of duplication of evolutionary importance have been met.

However, the initial, unique occurrence of a small duplication by unequal crossing-over is a problem, first, because it is extremely unlikely, given the nature of the process of recombination, which is not perfectly understood, and secondly because, as noted above, a regulatory mechanism of the kind proposed by Britten and Davidson (1969) implies that such duplications will be at a disadvantage, since they will be essentially nonfunctional, and yet will behave irregularly at meiosis. On the other hand, as Spofford (1969) has demonstrated, the process of incorporation is straightforward if an uncomplicated cistronic duplication confers an advantage equivalent to that of the heterozygote in a selectivity balanced polymorphism. Furthermore, if the frequency with which duplications occur is of the order of $10^{-7}$ or $10^{-8}$, then a reasonable assumption about the number of such sites (106, cf. Kimura's (1968) estimate of at least $10^{6}$ loci in mammals) suggests that the rate of incorporation will not be negligibly low (see table 1). Indeed, Nei (1969) has suggested that gene duplication must have occurred frequently, mainly by genome or chromosome doubling, and that this will lead to the presence of " many duplicate genes which have similar biological functions, and the function of one gene may be complemented by the other genes. If this is true, the effect of deleterious mutations or the so-called mutation load may be much reduced, and they may be expected to interact synergistically." If this were so, it would be difficult to account for the regularity of segregation of new mutations, or even of old ones. Nei also pointed out that the existence of repeated DNA sequences which vary greatly between closely related species (Walker, 1968) militates against his hypothesis. However, his conclusion that higher organisms may well be using only a small fraction of the total information stored in their DNA is important, since, for example, Britten and Davidson (1969) suggest a role for this kind of repeated DNA, yet their model, as noted earlier, does not fully explain other features of the possible evolution of duplication.

Spofford (1969) has also shown that her postulated mechanism of advantage to duplications would operate in haploids, but without the possibility of polymorphism developing, and thus might well have been a most important factor in allowing rapid early evolution before the development of stable control of ploidy.

\section{Summary}

The evolutionary consequences of duplications arising mainly by unique unequal crossing-over are considered, particularly with reference to rates of accumulation of duplications and the increased variability provided at meiosis by duplication.

Acknowledgments.-Much of this work was carried out while I held a C.S.I.R.O. Senior Overseas Studentship in the Institute of Animal Genetics, University of Edinburgh. I wish to thank Professor Alan Robertson, F.R.S., for helpful criticism.

\section{REFERENCES}

Allison, A. C. 1964. Polymorphisms and natural selection in human populations. Cold Spring Harbour Symp. Quant. Biol., 29, 137-149.

BAGLIONI, c. 1965. Abnormal hemoglobins. X. A study of haemoglobin Lepore Boston. Biochim. Biophys. Acta, 97, 37-46. 
BAXI, A. J., AND CAMOENS, H. 1969. Studies on haptoglobin types in various Indian populations. Human Heredity, 19, 65-70.

BRIDGES, C. B. 1935. Salivary gland chromosome maps. F. Hered., 26, 60-64.

BRITTEN, R. J., AND DAVIDSON, E. H. 1969. Gene regulation for higher organisms: a theory. Science, 165, 349-357.

CATCHeside, D. G. 1947. A duplication and a deficiency in Oenothera. F. Genet., 48, 99-110. FELLER, w. 1957. An Introduction to Probability Theory and its Applications. Vol. 1. New York, Wiley.

FIsHER, R. A. 1930. The Genetical Theory of Natural Selection (Revised 2nd Ed. 1958). New York, Dover.

FRASER, G. R., STEINBERG, A. G., Defaranas, B., MAYo, O., STAMATOYANNOPOUlOS, G., AND MOTULSKY, A. G. 1969. Gene frequencies at loci determining blood-group and serumprotein polymorphisms in two villages of N.W. Greece. Amer. F. Human Genet., 21, 46-60.

HALDANE, J. B. s. 1932. The Causes of Evolution. London, Longmans Green.

HALDANE, J. B. s. 1957. The conditions for coadaption in polymorphism for inversions. F. Genet., $55,218-225$.

HOPKINSON, D. A., SPENCER, N., AND HARRIS, H. 1964. Genetical studies on human red cell acid phosphates. Amer. F. Human Genet., 16, 141-154.

INGRAM, v. M. 1961. Gene evolution and the haemoglobins. Nature, 189, 704-708.

KIMURA, M. 1961. Natural selection as the process of accumulating information in adaptive evolution. Genet. Res., 2, 127-140.

KimURA, M. 1968a. Evolutionary rate at the molecular level. Nature, 217, 624-626.

KIMURA, M. 1968b. Genetic variability maintained in a finite population due to mutational production of neutral and nearly neutral isoalleles. Genet. Res., 11, 246-269.

KIMURA, M. 1969. The rate of molecular evolution considered from the standpoint of population genetics. Proc. Nat. Acad. Sci., 63, 1181-1188.

LEWTS, E. B. 1950. The phenomenon of position effect. Adv. Genet., 3, 73-117.

Lusi, I. E. 1966. The Biochemical Genetics of Vertebrates except Man. Amsterdam, North Holland Publ. Co.

MAYO, o. 1967. The use of simulation by computer in population genetics. Ph.D. thesis, University of Adelaide.

METZ, c. w. 1947. Duplication of chromosome parts as a factor in evolution. Amer. Nat., 80 , 81-103.

NAKAJIMA, H., TAKEMURA, T., NAKAJIMA, O., AND YAMAOKA, $x$. 1963. Studies on $\alpha$-methenyl oxygenase. F. Biol. Chem., 238, 3784-3796.

NANCE, W. E. 1963. Genetic control of hemoglobin synthesis. Science, 141, 123-130.

NEEL, J. V., SAlzano, F. M., JUNQUEIRA, P. C., KEITER, F., AND MAYBURY-LEWIs, D. 1964. Studies on the Xavante Indians of the Brazilian Matto Grosso.

NEI, M. 1969. Gene duplication and nucleotide substitution in evolution. Nature, 221, 40-42.

ohNo, s., MURAmoto, J., KLEIN, J., AND ATKIN, N. B. 1969. Diploid-tetraploid relationship in Clupeoid and Salmonid fish. In Chromosomes Today (eds. C. D. Darlington and K. R. Lewis). Edinburgh, Oliver and Boyd.

OHTA, T., AND KOJMA, K. 1968. Survival probabilities of new inversion in large populations. Biometrics, 24, 501-516.

ROGERS, N. G., BASNIGHT, M., GIBBS, C. J., AND GAJDUSEK, D. C. 1967. Latent viruses in chimpanzees with experimental kuru. Nature, 216, 446-449.

SCHWARZ, D., AND LAUGHNER, w. J. 1969. A molecular basis for heterosis. Science, 166, 626627.

smrrirEs, o. 1962. Chromosomal rearrangements and the evolution of haptoglobin genes. Nature, 196, 232-236.

smrrhIEs, o. 1964. Chromosomal rearrangements and protein structure. Cold Spring Harbour Symp. Quant. Biol., 29, 309-319.

spofrord, J. B. 1969. Heterosis and the evolution of duplications. Amer. Nat., 103, 407432.

STEPhEnS, s. G. 1951. Possible significance of duplication in evolution. Adv. Genet., 4, $247-$ 265.

sturtevant, A. H. 1925. The effects of unequal crossing over at the Bar locus. Genetics, 10, $117-147$.

WALKER, P. M. B. 1968. How different are the DNAs from related animals? Nature, 219, 228-232.

WATTS, R. L., AND WATTS, D. c. 1968. The implications for molecular evolution of possible mechanisms of primary gene duplication. F. Theoret. Biol., 20, 227-244. 
Weatherall, D. J. 1969. The genetics of thalassaemias. Brit. Med. Bull., 25, 24-29.

WHTtEHOUSE, H. L. к. 1965. Towards an Understanding of the Mechanism of Heredity. London, Edward Arnold.

WORKMAN, P. L. 1968. Gene flow and the search for natural selection in man. Human Biol., 40, 260-279.

ZIMMERMAN, F. K., AND GUNDELACH, E. 1969. Intragenic complementation, hybrid enzyme formation and dominance in diploid cells of Saccharomyces cerevisae. Mol. Gen. Genet., 103, 348-362.

ZUCKERKANDL, E., AND PAULING, L. 1965. Evolutionary divergence and convergance in proteins. In Evolving Genes and Proteins (eds. V. Bryson and H. J. Vogel). New York, Academic Press.

\section{NOTE ADDED IN PROOF}

Recent work (Jones and Robertson, 1970; Southern, 1970) suggests that highly reiterated DNA is unlikely to code for proteins, but is more likely to be important in chromosomal and, particularly, centromeric localisation; this makes Britten and Davidson's findings even more open to question and re-evaluation than has been emphasised above.

JONES, K. W., AND ROBERTSON, F. W. 1970. Localisation of reiterated nucleotide sequences in Drosophila and mouse by in situ hybridisation of complementary RNA. Chromosoma, 31, 331-345.

sourHern, E. 1970. Base sequence and evolution of guinea-pig $\alpha$-satellite DNA. Nature, 227, 794-798. 